\title{
Designing upper-division thermal physics assessment items informed by faculty perspectives of key content coverage
}

\author{
Katherine D. Rainey $\odot$, Michael Vignal, and Bethany R. Wilcox \\ Department of Physics, University of Colorado, 390 UCB, Boulder, Colorado 80309, USA
}

(Received 25 May 2020; accepted 27 July 2020; published 26 August 2020)

\begin{abstract}
Though several conceptual inventories have been developed for thermal physics, none target upperdivision material and all focus specifically on thermodynamics without including statistical mechanics content. In this paper, we outline the development process of an upper-division thermal physics assessment that captures both thermodynamics and statistical mechanics content. To ensure that the assessment can be broadly usable, as a first step in this process, we administered a survey to physics faculty to determine the scope and content variability of thermal physics courses across institutions. We received over 70 responses from 63 unique institutions, approximately half of which are minority-serving institutions and women's colleges. Our findings support the claim that there is significant variation in the content covered at different institutions, but also some general agreement on a number of core content areas. We identified 10 key topics which were listed by the majority (95\%) of survey respondents to focus on for the assessment development process. We then wrote assessment objectives that encompass core content goals within these 10 topics, which guided the writing of free-response assessment items that were piloted with students. Using student responses to the free-response items, we developed multiple-response versions, which includes both multiple-choice and coupled, multiple-response items. In this paper, we present details of the faculty survey, including methods of developing and distributing the survey to solicit a broad range of perspectives. Additionally, we present results of the survey, including core content covered by faculty in upper-division thermal physics courses, and discuss how these results were used to guide development of assessment objectives and assessment items. We include the full development process of one assessment item as an example.
\end{abstract}

DOI: 10.1103/PhysRevPhysEducRes.16.020113

\section{INTRODUCTION}

Thermal physics, which includes both thermodynamics and statistical mechanics, is a core course required for attaining a physics bachelor's degree at most institutions. However, a dearth of validated assessments in the realm of upper-division thermal physics presents challenges in measuring student understanding of this content to inform course transformations. In order to improve course instruction and student outcomes, researchers and instructors must first have some method of evaluating what students know and are able to do with that knowledge.

Creating an assessment that can provide feedback to guide instructors is a challenge for upper-division thermal physics. Anecdotally, the material covered in upperdivision thermal physics courses often varies between instructors and across institutions. For example, some teach

Published by the American Physical Society under the terms of the Creative Commons Attribution 4.0 International license. Further distribution of this work must maintain attribution to the author(s) and the published article's title, journal citation, and DOI. thermodynamics, others teach statistical mechanics, and still others teach a combination of both (which we refer to as "thermal physics"). Additionally, some topical areas may not be prioritized by every instructor teaching thermal physics (e.g., diffusion or temperature-entropy diagrams). This content variability, in addition to notational differences across thermal physics texts, poses a significant challenge in the development of standardized thermal physics assessments and teaching tools that can be utilized by a wide range of instructors. Therefore, an essential first step in creating a thermal physics assessment is to determine what thermal physics content is taught at most institutions.

In this paper, we discuss the first stages of the process used in developing an upper-division thermal physics assessment. This process will be unique in 3 ways. First, it will have taken into account the diverse perspectives of over seventy instructors (at various types of institutions) with respect to content priorities in their upper-division thermodynamics and/or statistical mechanics courses. Second, it will involve novel efforts to create coupled, multiple-response items [1] that address scientific practices, which have become more integral to science education and assessment since the introduction of the Next Generation Science Standards for K-12 grade levels [2]. Third, the 
assessment development process described in this paper will lay the foundation for an eventual flexible assessment allowing for customization of included content, which will be developed as part of future work.

After reviewing relevant background information (Sec. II), we describe the process of constructing and distributing a faculty survey to solicit content priorities for upper-division thermodynamics and/or statistical mechanics courses (Sec. III). We present results of the faculty survey, including general course information, key concepts covered, and valued scientific practices. We also compare between survey responses and submitted syllabi as a reliability check on the survey, and present an analysis of content variability across institutions. These survey results informed the development of assessment items for upper-division thermal physics (Sec. IV), consistent with the material presentation of the most common textbook. We then discuss future work for development of a standardized assessment (Sec. V). We conclude with a synopsis of the assessment development process thus far and reflect on that process's possible application to other assessments (Sec. VI).

\section{BACKGROUND}

Assessments that address students' conceptual understanding of physics play an important role for both physics educators and physics education researchers. They can be used by educators to measure the impact of instructional approaches or curricular changes. For example, the Force Concept Inventory (FCI) [3] has been used for multiple introductory physics investigations since its initial development [4]. In physics education research, assessments such as these are often an outcome of investigations into student reasoning and used to investigate student understanding of physics concepts.

Education research studies on thermal physics content, in the realms of biology, chemistry, engineering, and physics, are becoming increasingly present in the literature $[5,6]$. These studies have utilized isolated thermal physics problems, small quizzes, and larger-scale assessments to investigate student conceptual understanding of primarily introductory thermal physics topics. Investigations of upper-division thermal physics content are comparatively less common than work at the introductory level. Additionally, there is currently no upper-division physics assessment that includes both thermodynamics and statistical mechanics. Existing thermal physics assessments instead focus on classical thermodynamics topics, such as heat, temperature, and thermodynamic laws [7-11]. At the current time, we are unaware of any statistical mechanics assessments. A lack of a fully encompassing, upperdivision thermal physics assessment leaves a gap in the ability of professors and researchers to test upper-division students' understanding of higher-level thermal physics concepts (e.g., statistical mechanics) and facilitate further investigations of student understanding at the upperdivision level. In this section, we briefly review research on student difficulties in thermal physics and the status of assessment in physics, highlighting thermal physics assessment. We also include a discussion of validation methods, which informed our approach in soliciting participants when designing the assessment.

\section{A. Student content understanding}

There have been many studies regarding thermal physics that have focused on student understanding of thermodynamics content in physics, biology, and chemistry [5]. Several studies across these disciplines have found students have particular difficulties distinguishing between heat and temperature [12-14]. Other investigations have looked at student difficulties with entropy [15-18], appropriate applications of the ideal gas law [19,20], and energy in the context of the first law of thermodynamics [21,22]. Most of these studies have investigated student conceptual understanding in the realm of introductory thermodynamics content, mainly at the high school and introductory-college level [5].

Upper-division investigations are comparatively less common, especially for statistical mechanics, though some exist. Several examples of foci for these few investigations include student reasoning surrounding the Boltzmann factor [23] and Taylor expansions in statistical mechanics [24], as well as some statistical mechanics instructional methods, such as using statistical approaches to teach entropy [25]. Some researchers have investigated student reasoning in thermal physics through interviews, reviews of student coursework, or conceptual assessments, while others have utilized tutorials. In particular, Smith et al. created tutorials to investigate student reasoning using the Boltzmann factor to compare relative probabilities of states [23] and to address students' conceptions of entropy when approaching Carnot cycles [15]. We utilized a subset of questions from these tutorials to inform item development for our assessment.

\section{B. Assessment in physics}

Though some lower-division thermal physics assessments exist, there is still a need for a standardized upperdivision thermal physics assessment that includes both thermodynamics and statistical mechanics concepts. Currently, there are six assessments categorized as "thermal or statistical" assessments on PhysPort, an online repository of PER-based resources for physics faculty. ${ }^{1}$ However, all of these assessments are categorized as being for "intro college" or "intermediate" levels (i.e., not upper-division)

\footnotetext{
${ }^{1}$ We do not include a link to PhysPort, as it is susceptible to change. PhysPort can be found via an online search engine. The full webpage title is "PhysPort: Supporting physics teaching with research-based resources."
} 
and, based on the four assessments that are readily available online [7,9-11], none cover statistical mechanics concepts. We note that only five of these six thermal physics assessments were finalized and the sixth was recommended to not be used by the assessment developer. We did not receive a response from the developer of one of the completed five assessments and thus could not access it.

All four assessments accessible through PhysPort ${ }^{2}$ were developed based on research on student thinking, were studied and implemented at multiple institutions, and resulted in peer-reviewed journal articles and a dissertation [7-11]. The development cycle of these assessments is not discussed in most of these publications. However, Brown's dissertation outlines the development of the most recent thermal physics assessment on PhysPort - the Survey of Thermodynamic Processes and First and Second Laws (STPFaSL), an assessment that we referenced during our item development process [11]. The process the STPFaSL developers took aligns with the historical approach taken for developing other assessments, included identifying focus topics (via course learning goals) and iterative question development and refinement based upon expert feedback, student interviews, and classroom testing [26].

Most PER-based assessments, including existing thermal physics assessments, have intentionally narrow scopes and hone in on a very specific subset of topics within a particular subdiscipline of physics. Some of the very first conceptual physics assessments, such as the FCI [3], have done this, and other assessments have followed suit. The narrow scopes of these assessments makes them more useful for a broad range of practitioners who need to identify specific conceptual difficulties to inspire pedagogical changes. Additionally, narrow scopes can be motivated by assessment validation restrictions, as discussed in Sec. II C. The scope of existing thermal physics assessments includes concepts such as specific thermodynamic laws and processes [11], as well as basic thermodynamic concepts such as heat and temperature [7-9], phase transformations [9], and thermal properties of materials $[9,10]$. The scopes of the four accessible thermal physics assessments do not include statistical mechanics concepts.

\section{Assessment validation}

From the beginning of our development process, we considered possible validation approaches for the assessment. In particular, we considered two validation approaches (i.e., classical test theory and item response theory). One of these (item response theory) can assist in identifying bias within assessment items, which results in differential

\footnotetext{
${ }^{2}$ We were able to access the Heat and Temperature Conceptual Evaluation (HTCE), the Survey of Thermodynamic Processes and First and Second Laws (STPFaSL), the Thermal Concept Evaluation (TCE), and the Thermodynamic Concept Survey (TCS).
}

performance between individuals of the same ability but different backgrounds (e.g., gender or race) [27]. With an eye towards reducing bias in our assessment, we began taking diverse perspectives into account early on. For example, we solicited faculty perspectives from a diverse range of institutions and departments, many of which may often be excluded from large-scale assessment studies. This is due to the fact that these studies typically require large student populations and thus large research institutions, which tend to be predominately white, are often targeted. Inclusion of multiple faculty perspectives allows us to expand the scope of the assessment while still making it broadly applicable for many instructors.

As mentioned in Sec. II B, narrow content scopes within assessments can provide more pinpointed insights into student difficulties in a specific content area. However, the need for specific content areas to target in pedagogical reform is not the only motivation for these limited scopes. The narrow scopes within assessments can also be attributed to content variability across institutions (especially at the upper-division level) and the restrictions of many classical test theory (CTT) validation requirements, such as unidimensionality-the idea that an assessment's scope must focus on one construct (e.g., focusing on "forces" or "motion," as opposed to "introductory physics").

CTT is a commonly used approach for validation of conceptual assessments in education. One advantage of CTT that has made it so commonly used is its theoretical assumptions, which make it easily applicable to different testing situations. Despite its advantages, CTT also has some drawbacks. The CTT requirement of unidimensionality limits the scope of content that can be included on an assessment. Thermal physics spans a large space ranging from classical thermodynamics to statistical mechanics. A thermal physics assessment that captures both classical thermodynamics and statistical mechanics may or may not be considered unidimensional, as it would test more than one core topic or idea.

In addition to unidimensionality, there is a more fundamental issue at the heart of CTT that has led to problematic outcomes in scores between different groups of students. This issue comes from fundamental assumptions of CTT: the validation statistics, and therefore the reliability and validity, are determined by the group of students whose results were used in the initial validation [28]. This population dependence of validation statistics makes it difficult to compare assessment performance between different groups of students.

Results of some CTT-validated assessments have found "achievement gaps," particularly between women and men [29-32]. In these studies, men tend to perform better on these assessments than women. This could lead an unwary reader to conclude that men are more capable of performing well in physics than women. However, due to the demographic composition of physics majors, which is 
predominately male (and white) [33], it is likely that these assessments were implemented in predominately white and male departments. This means that the group-dependent statistics resulting from CTT use are normalized to a group of mostly white men. It may well be that the explanation for these "achievement gaps" is not the result of inherent differences between genders, but instead are a result of the population-dependent nature of the approach used for validation.

To avoid potential bias within assessment items, researchers can invoke different validation methods that mathematically separate "student ability" 3 and difficulty. One approach is one-dimensional item response theory (IRT), known as the Rasch model. The mathematics of the Rasch model separate parameters describing student ability from the difficulty of items. The Rasch model assumes student performance on an assessment item is based solely on their ability and the item difficulty [34]. A result of this model is that these parameters are not dependent on the population used to validate the assessment. This reduces the potential for bias in the assessment and makes comparisons across institutions susceptible to less ambiguity.

When our upper-division thermal physics assessment is piloted at a larger scale, we plan to explore the Rasch model for validation statistics. This will require a large set of students $(N \geq 100)$. It will also require a diverse set of students in order to make claims about performance differences across different populations and try to reduce test bias. To aid in this endeavor, we solicited survey respondents (discussed in Sec. III) from a wide range of institutions, including large and small departments as well as minority-serving institutions and women's colleges. In the survey, respondents could indicate interest in piloting for the large-scale implementation.

With potential validation methods in mind, we began the process of developing our assessment by first probing the physics community about content priorities in upperdivision thermal physics courses. We did this by constructing and distributing a survey to physics faculty, as discussed in Sec. III.

\section{THE FACULTY CONTENT SURVEY}

It is commonly suggested that content coverage in upperdivision courses can vary significantly between different physics departments and even different instructors within the same department. This poses a significant challenge in developing an assessment that will be useful for a broad range of instructors and courses. For example, if an

\footnotetext{
${ }^{3}$ We note the term student ability is the term used historically in the literature and refers to a statistical output of validation models; it is a measure of performance as opposed to innate ability of individuals. We use this historically utilized term for consistency with the existing literature. However, we acknowledge the potentially problematic nature of this term.
}

assessment contains even a single question related to content not covered in an instructor's course, the assessment will likely be less useful for that instructor or not be used by that instructor at all. There are two strategies for addressing this issue. One is to create an assessment that can be customized to different courses, allowing topics present on the assessment to be tailored for specific courses. The other is to create an assessment that only touches on topics covered by the majority of instructors. For our thermal physics assessment, we began by attempting the latter, with the aim of working toward a customizable assessment in the future.

In an effort to inform content foci for assessment development, we developed and distributed a content survey to be completed by physics faculty familiar with teaching upper-division thermal physics. The survey was distributed to a diverse range of institutions and designed to solicit key information about thermal physics courses, such as content covered, general course structure and emphasis (thermodynamics, statistical mechanics, or both), and needs or interest in an upper-division thermal physics assessment. This section describes methods for developing and distributing the survey with an emphasis on creating a format that was accessible and relatively short in duration, while still soliciting sufficient information. We present results from the survey, including texts utilized and content foci in upper-division thermal physics courses.

\section{A. Survey development}

Prior to constructing the faculty survey, a focus group was conducted with four experts, all with experience teaching thermal physics and/or researching student difficulties in thermal physics. The focus group solicited expert perspectives surrounding upper-division thermal physics, including textbooks, content coverage, learning goals, and existing thermodynamics assessments. Outcomes from the focus group informed several questions included on the faculty survey. For example, participants discussed notational conventions as one major challenge for a thermal physics assessment (e.g., the sign convention of work). To address this concern, one question on the survey solicited specific notational issues worth considering in development of a thermal physics assessment. Additionally, textbooks brought up during the focus group comprised the list of textbook options provided on the survey.

To facilitate ease of responses, the faculty survey was a primarily multiple-response format with only a select set of questions being free response. Thus, one of the first steps in survey development was determining which options to provide for various multiple-response questions. We began by investigating the scope of thermal physics in texts; we analyzed six thermal physics texts brought up during the focus group [35-40] for key content coverage. This process involved reviewing each text and identifying topical areas for each based on chapter titles, section headings, and emphasized key terms. Based on the frequency of topics 
appearing across the different texts, we classified topical areas into core topics and supporting topics. To put these into an accessible form for use in the survey, topics were sorted and condensed into 29 core topics, most with roughly 4 supporting topics (see Sec. III C). For example, the core topic of "thermodynamic laws" had four supporting topics: 0th law, 1st law, 2nd law, 3rd law. Some core topics had no supporting topics (e.g., semiconductors) while some had as many as seven (e.g., energy and thermodynamic potentials); the one exception to this was statistical mechanics, which had 14 supporting topics.

In addition to focusing on concepts, the survey also solicited information on the scientific practices valued by respondents in their thermal physics courses. The list of scientific practices provided on the survey was pulled from the Next Generation Science Standards (NGSS) list of science and engineering practices [2]. In this list, similar practices are combined together (e.g., developing and using models); however, in upper-division courses, it is less clear that all paired practices would be targeted together. Thus, to collect more specific data about individual practices, paired NGSS practices were split into separate categories. For example, "developing and using models" was split into "developing models" and "using models" for the survey.

The survey was administered online ${ }^{4}$ and divided into 4 major sections:

(1) General course information

(2) Content coverage

(3) Scientific practices

(4) Interest in, and concerns about, an upper-division thermal physics assessment

In addition to providing general course information in section (1), respondents also had the option to identify their institution and submit their course syllabus. Finally, gender and racial identity information were collected at the end of the survey, after section (4).

After initial construction of the survey, we solicited feedback from physics faculty at the University of Colorado Boulder who were familiar with teaching upper-division thermal physics. Based on these discussions, and informed by the frequency of topical areas appearing across the six different analyzed texts, we grouped the core topics into two categories: assumed core topics and other core topics. Assumed core topics are topics that one might expect are covered in every thermal physics course. In alphabetical order, the assumed topics were

- Energy and thermodynamic potentials

- Engines and refrigerators

- Entropy

- Equilibrium

- Monatomic gases

- Heat

\footnotetext{
${ }^{4}$ The survey was administered through the survey platform Qualtrics and hosted by the University of Colorado Boulder (CU).
}

- Temperature

- Thermodynamic laws

- Work

The survey presented these assumed core topics at the beginning of section (2) of the survey, with their supporting topics shown on the same page. A free-response text box followed these assumptions to allow respondents to indicate disagreement with the assumptions made. All other core topics were provided on the following page of the survey without their supporting topics displayed. After selecting from the list of other core topics, associated supporting topics for each of the selected core topics were displayed on the following page (while all other supporting topics associated with core topics not selected were not displayed). This conditional formatting was motivated by the desire to reduce respondent fatigue due to survey length.

\section{B. Survey distribution}

To ensure the information collected was reflective of a broad range of institutions, we collected contact information for a large variety of physics degree-granting institutions, including minority serving institutions (MSIs) and women's colleges, for use in distributing the survey. Institutions were identified using the American Physical Society's "Top Educators" lists [42], each of which identifies 16-20 institutions with the highest average number of physics bachelor's degrees awarded by the institution per year, sorted by highest physics degree offered at each institution. We utilized the overall and underrepresented minority (URM) lists for Ph.D.-granting, MS-granting, and BS-granting institutions. Beyond that, we used the American Physical Society's MSIs list [43], to identify all other physics-degree-granting MSIs not on the Top Educators lists; the MSI list included institutions with both large and small physics departments. This list identifies Historically Black Colleges and Universities, Black-serving institutions, Hispanic-serving institutions, and institutions serving Asian Americans and Native American Pacific Islanders that offer degrees in physics. We also identified women's colleges with the "Women in Physics" report produced by the American Institute of Physics [44].

After identifying institutions, we obtained contact information of department chairs from physics department websites. We then emailed the survey solicitation to the department chairs, with a specific request for the email to be forwarded to all faculty within their department who were currently teaching or had previously taught upperdivision thermal physics. In addition to department chairs, the research team solicited the help of their professional contacts at different institutions to take the survey or forward it to faculty in their department.

After initial solicitations were sent and data were collected, we saw that a large geographic region had not 
been captured by our data. This prompted us to identify institutions in geographic areas within the United States that were not captured by the Top Educators, MSI, or women's colleges lists using the PhysNet directory of U.S. Physics Institutions [45]. The survey was reopened and a second round of emailed solicitations were sent to newly identified departments. In this second round of solicitations, we received 18 new respondents, largely from states that were not represented initially.

\section{Survey results}

The survey was open for response collection for three and a half months, then opened again a few months later for an additional month and a half. During these time windows, 73 respondents fully completed the survey while 2 completed all of the survey except questions regarding scientific practices (Sec. III of the survey) and interest in assessment (Sec. IV). Only responses that completed the sections with core topics and supporting topics (Sec. II) or beyond were used for analysis. We do not report response rate, as it is unclear how many people received the solicitation forwarded from their department chairs. We estimate between 200 and 250 emailed solicitations were sent.

Figure 1 shows locations of responding institutions; many geographic regions across the United States are represented in our sample, with the exception of the north central region. Overall, we identified 63 unique institutions from the survey, 32 of which were MSIs and/or women's colleges; 2 institutions could not be identified. Figure 2 presents institution type by highest physics degree offered and MSI or women's college classification. In a few cases, $(N=7)$ institutions were represented by $2-3$ responses; it was evident from submitted syllabi and individual item responses that these were submitted by different people.

Racial demographics of respondents included Asian $(14 \%, N=10)$, Black/African American $(1 \%, N=1)$, Caucasian $(76 \%, N=55)$, and Hispanic $(4 \%, N=3)$;

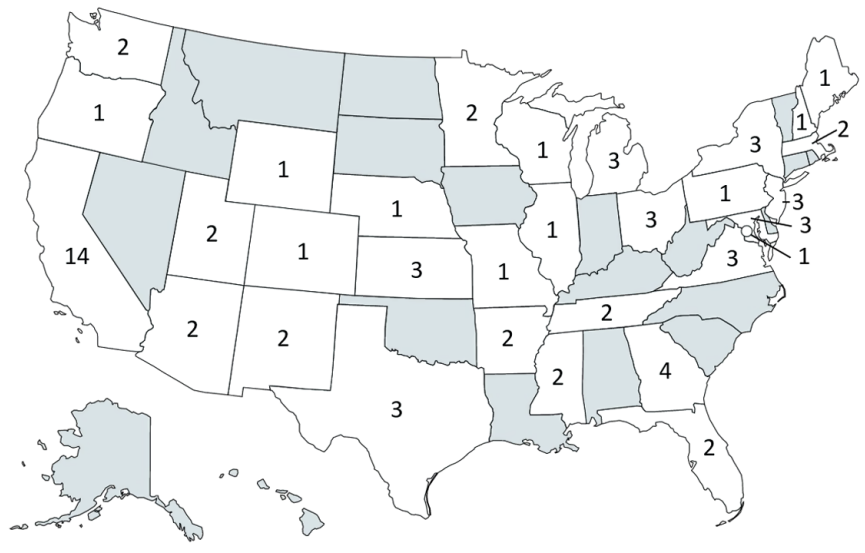

FIG. 1. Number of survey responses per state. Darker gray states had no respondents. One additional response was received from an international location not displayed in the map.

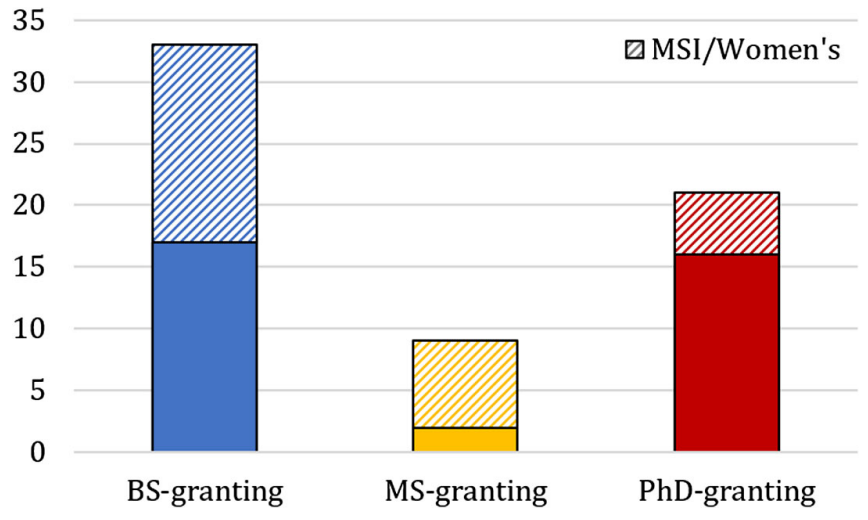

FIG. 2. Highest physics degree offered by institution classification. Minority-serving institutions (MSIs) or women's colleges are indicated in striped boxes and other institutions are presented in solid colors. Institutions granting bachelor's degrees (BS), master's degrees (MS), and Ph.D.s are indicated.

no other racial identities were indicated and $6 \%(N=4)$ preferred not to answer. Additionally, $85 \%(N=61)$ of respondents were men and $13 \%(N=9)$ were women (no other gender identities were indicated); $3 \%(N=2)$ preferred not to provide their gender. Three respondents did not provide any demographic information.

We collected institutional information, including selectivity, research activity, student population, and highest physics degree offered via the Carnegie classifications [46] and institutions' physics department websites. From the Carnegie classifications, we identified $73 \%(N=45)$ of identifiable institutions as being selective or more selective with regards to admissions practices, while $27 \%(N=17)$ are considered "inclusive" institutions. Additionally, 54 schools are classified as having high or very high research activity. We note that one institution was not in the Carnegie classification database, as it was not an institution in the United States.

\section{Course information}

We asked respondents if their course focused on thermodynamics, statistical mechanics, or both (thermal physics); 95\% $(N=71)$ selected thermal physics and the remaining $5 \%(N=4)$ of responses were split evenly between thermodynamics and statistical mechanics. Most institutions reported one semester of thermal physics $(80 \%$, $N=59)$; some reported two quarters $(7 \%, N=5)$ or two semesters $(8 \%, N=6)$, while a small minority reported one quarter $(4 \%, N=3)$. Respondents reported that their courses were composed of mostly juniors $(N=56)$ and seniors $(N=49)$, though some $(N=12)$ reported sophomores in the course as well. Respondents could choose multiple student populations for this portion of the survey.

The majority of respondents $(69 \%, N=52)$ reported using An Introduction to Thermal Physics by 


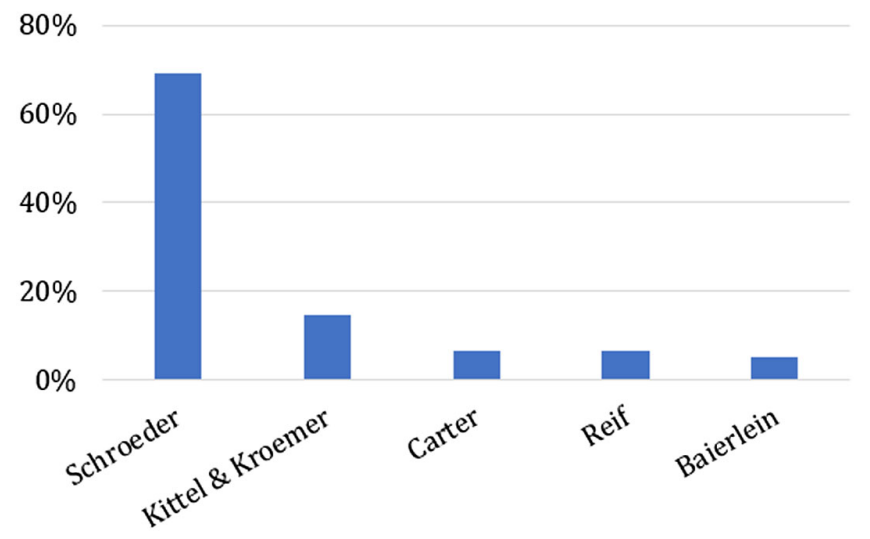

FIG. 3. Most common textbooks reported being used in upperdivision thermal physics courses [35-37,39,47]. Respondents could select multiple textbooks on the survey or indicate using another textbook not on the provided list, or none at all. Thus the percentages do not sum to $100 \%$.

Schroeder [39]. Thermal Physics by Kittel and Kroemer [37] was the second most frequently cited text $(15 \%$, $N=11$ ). All other texts appeared at a frequency of $7 \%$ or below. Figure 3 shows frequencies of the most common textbooks. We note $5 \%$ reported using no textbook and $27 \%$ reported using other texts not provided on the survey, though none of these other texts appeared at frequencies over 5\%. Many of the instructors $(40 \%, N=30)$ indicated they teach with the assumption that their students have no prior exposure to thermal physics content. Some expected familiarity with topics such as energy, heat, the first and second laws of thermodynamics, and the ideal gas law. A few $(N=11)$ said they expect thermal physics exposure from the introductory physics sequence, though several noted that thermal physics is only covered for a few weeks, and sometimes not at all, in that sequence.

Overall, these data show most institutions require one semester of thermal physics, most instructors use Schroeder's text [39], and many instructors assume their students have no prior exposure to thermal physics content. These results suggest two implications for physics education research: (i) development of Schroeder-based thermal physics assessments and materials could serve many instructors and institutions, though would still exclude the sizable population of instructors and institutions who do not use that text; and (ii) pretest administration of the upper-division thermal physics assessment we develop may not produce meaningful measurements of student understanding of thermal physics content prior to taking the course.

\section{Key topical areas}

Table I shows frequency of assumed core topics, assumed core topics' supporting topics and, other core topics. Most assumed core topics (see Sec. III A) appeared at a frequency of $100 \%$. In two responses, no supporting topics were selected under one of the nine assumed core

TABLE I. Topic frequency from faculty content survey. The two left columns show data for assumed topics. All assumed core topics appeared at a frequency of $100 \%$ with the exception of engines and refrigerators. Frequencies for engine and refrigerator supporting topics are calculated using the total number who selected that core topic $(N=71)$. The rightmost column shows all other core topics. Frequencies above 95\% appear in bold. See Supplemental Material [41] for other core topics frequencies. Topics that appeared on syllabi but not the survey (e.g., ensembles and thermodynamic identities) are also not presented.

\begin{tabular}{lrlrl}
\hline \hline Assumed core (and supporting) topics & $\%$ & Assumed core (and supporting) topics & $\%$ & Other core topics \\
\hline Energy and thermodynamic potentials & $\mathbf{1 0 0}$ & Equilibrium & $\mathbf{1 0 0}$ & Statistical mechanics \\
Chemical potential & $\mathbf{9 5}$ & Thermal equilibrium & $\mathbf{9 9}$ & Processes \\
Energy sources & 47 & Stable and unstable equilibrium & 44 & Fermions \\
Enthalpy & 88 & Heat & $\mathbf{1 0 0}$ & Bosons \\
Equipartition & $\mathbf{9 6}$ & Heat capacity & $\mathbf{1 0 0}$ & Blackbody radiation \\
Free energy (Gibbs and Helmholtz) & $\mathbf{9 6}$ & Heat transfer & 72 & Phases \\
Internal energy & $\mathbf{9 7}$ & Latent heat & 91 & Diatomic gases \\
Maxwell's relations & 80 & Temperature & $\mathbf{1 0 0}$ & Quantum phenomenon \\
Engines and refrigerators & $\mathbf{9 5}$ & Absolute zero & $\mathbf{9 7}$ & Kinetic theory \\
Heat engines & $\mathbf{9 7}$ & Negative temperature & 65 & Pressure diagrams \\
Refrigerators & 87 & Thermodynamic temperature & 88 & Scaling \\
Entropy & $\mathbf{1 0 0}$ & Temperature measurement & 57 & Magnetism \\
Boltzmann's law & 92 & Thermodynamic laws & $\mathbf{1 0 0}$ & Chemical reactions \\
dS dQ/T & $\mathbf{9 5}$ & Oth & 89 & Solids \\
Entropy and information & 56 & 1st & $\mathbf{1 0 0}$ & Conduction, convection, radiation \\
TS diagrams & 71 & 2 nd & $\mathbf{1 0 0}$ & Pure substances \\
Gases & $\mathbf{1 0 0}$ & 3rd & 88 & Diffusion \\
Ideal gas law & $\mathbf{1 0 0}$ & Work & $\mathbf{1 0 0}$ & Cooling techniques \\
Mixtures of gases & 60 & Mechanical & $\mathbf{9 9}$ & Fluids \\
van der Waals interactions & 71 & Path dependence & 89 & Semiconductors \\
\hline \hline
\end{tabular}


topics. However, in neither of these instances was it indicated in the provided text box that the respondent disagreed with the assumption that they cover that topic. The one assumed core topic that did not appear at $100 \%$ frequency (engines and refrigerators) was selected by only $95 \%$ of respondents.

Table I shows the frequency of supporting topics relative to the number of times the corresponding core topic was selected, and the frequency of core topics relative to the total number of valid responses (i.e., responses completed through Sec. II of the survey). We present frequencies of all other core topics. See Supplemental Material [41] for presentation of the 56 other supporting topics and frequencies.

We found that all 9 of our assumed core topics, as well as statistical mechanics, are covered by at least $95 \%$ of respondents in their upper-division thermal physics courses. These results are relevant for all researchers interested in materials and assessment development in upper-division thermal physics. For the purpose of developing an upper-division thermal physics assessment, we used these results to prioritize content foci for assessment item development. Using these results as a baseline helps in creating an assessment that can serve a wide range of instructors and institutions.

\section{Scientific practices}

Of the 16 practices presented on the survey, three appeared at a frequency of over $85 \%$ : using mathematical thinking $(99 \%, N=72)$, asking questions $(97 \%, N=71)$, and using models $(89 \%, N=65)$. Review of syllabi indicates the practice of "asking questions" may have been misinterpreted by survey respondents; the NGSS practice refers to asking scientific questions (namely, for scientific investigations), but we suspect many respondents may have interpreted this practice as referring to asking questions about content during class or office hours. The next most frequently appearing practice was constructing explanations $(71 \%, N=52)$, while the remaining 12 practices appeared at a frequency of $64 \%$ or less, as can be seen in Table II.

These results highlight four scientific practices that stand out as valued by a large majority of thermal physics instructors in our sample: using mathematical thinking, asking questions, using models, and constructing explanations. The data demonstrate many other scientific practices are less of a universal focus for thermal physics courses at the upper-division level. Thus, we paid particular attention to including opportunities for students to demonstrate engagement in the practices of using models, using mathematical thinking, and constructing explanations within some developed assessment items. (We exclude "asking questions" here because it may have been misinterpreted by respondents and would be difficult to assess on a multiple-response assessment.)

\section{Assessment concerns and interest}

Beyond collecting information about upper-division thermal physics courses, the survey also included questions directly related to the assessment. In particular, we asked about potential notational issues they could see as worthy of consideration in the assessment design and faculty interest in the assessment, including how they would use it and if they would be interested in piloting the multipleresponse assessment in their upper-division thermal physics courses.

Notational concerns.-Of all respondents, 29 faculty provided possible notational concerns on the survey. The survey question about notational concerns gave the sign convention of work as an example, and 13 respondents indicated in their response to this open-ended question that the sign of work would be a concern for assessment development; this was the most common concern cited. Others mentioned commonly used symbols (e.g., $k$ vs $k_{B}$ ); the sign of work's impact on the form of the first law of thermodynamics (e.g., $\Delta U=Q+W$ vs $\Delta U=Q-W$ ); the sign convention of heat for certain applications (e.g., engines); and units (e.g., some texts use unitless quantities whereas others do not). Informed by these notational concerns, and knowing which texts are used the most frequently, we have largely utilized the notation used in Schroeder's text [39] when developing assessment items, and have clearly defined all symbols within assessment items or at the beginning of the assessment. We avoided potential confusion about the sign of work and heat by

TABLE II. Frequencies of valued scientific practices valued by physics faculty in upper-division thermal physics. Frequencies were determined using the number of respondents who completed through Sec. III of the faculty content survey $(N=73)$. The practices were taken from the NGSS list of science and engineering practices [48].

\begin{tabular}{|c|c|c|c|c|c|}
\hline Scientific practice & $\%$ & Scientific practice & $\%$ & Scientific practice & $\%$ \\
\hline Using mathematical thinking & 99 & Interpreting data & 59 & Developing models & 45 \\
\hline Asking questions & 97 & Defining problems & 58 & Obtaining information & 40 \\
\hline Using models & 89 & Using computational thinking & 58 & Designing solutions & 38 \\
\hline Constructing explanations & 71 & Analyzing data & 51 & Carrying out investigations & 12 \\
\hline Communicating information & 64 & Engaging in argument from evidence & 47 & Planning investigations & 10 \\
\hline Evaluating information & 62 & & & & \\
\hline
\end{tabular}


asking which direction work made energy flow or which direction heat flowed (e.g., into or out of the system). We also only asked for the magnitude of work when looking for symbolic or numeric responses related to work.

Faculty interest in assessment.-The survey included one question regarding faculty interest in the assessment, specifically asking if and how faculty would use the assessment once completed. The most common use cited by respondents was using the assessment to measure student learning in their course $(N=13)$, often with pre-post administration $(N=11)$. Others said they would be interested in using it to inform their teaching strategies $(N=12)$ and compare student learning in their course with other courses $(N=11)$. A few $(N=4)$, said they would use the assessment to inform content or tracking student progress. Of the 43 respondents who answered this question, none indicated they did not see obvious value in the assessment. However, in interpreting respondents' sense of value, it is important to note this group was composed of a self-selected set of faculty that chose to complete the survey.

\section{Response consistency}

As a verification of the survey data, we checked for consistency between survey responses and submitted syllabi for the 51 responses that provided a syllabus. We looked at key topics on syllabi and compared with the associated survey response to ensure topics appearing on the syllabus also appeared on the survey response. Here, we refer to "discrepancies" as a mismatch between topics on the syllabus and those selected on the survey (e.g., topics appear on the syllabus but were not selected on the survey). The topic of diffusion was listed by five respondents in their syllabus and not their survey responses. No other core or supporting topics had more than 3 discrepancies when comparing between survey responses and the syllabus.

Discrepancies could be due to the amount of focus placed on those topics in the course. For example, BoseEinstein condensates may appear on the syllabus but may not be seen as a major content focus for the instructor when completing the survey, resulting in a discrepancy between their syllabus and response. Some topics, such as interacting systems $(N=12)$, ensembles $(N=13)$, large systems $(N=16)$, and Boltzmann and/or quantum statistics $(N=17)$, appeared in syllabi but did not appear as explicitly named core or supporting topics on the survey. However, those who included topics such as these on their syllabus selected other topics on the survey that encompass or require the same idea, such as multiplicity, thermal equilibrium, and specific statistical distributions. This comparison shows that the survey reliably captured the scope of content coverage for most survey responses without large discrepancies.

\section{Content variability}

To investigate the claim of content variation across upper-division thermal physics courses, we examined survey responses to see how many topics were selected by all instructors. We looked at the three groups of topics laid out in Table I: assumed core topics, assumed core topics' supporting topics, and other core topics.

We found that $9 / 9(100 \%)$ of assumed core topics, $11 / 32(34 \%)$ of assumed supporting topics, and $2 / 20$ $(10 \%)$ of other core topics were selected by at least $90 \%$ of respondents. When looking at the $100 \%$ threshold, we found that 8/9 (89\%) of assumed core topics, 4/32 (13\%) of assumed supporting topics, and 0/20 (0\%) of other core topics were selected by all respondents. These results suggests that there is little alignment (outside of our assumed core topics) across instructors and institutions. This lack of alignment holds true even within an institution, though to a lesser extent: at institutions with multiple survey respondents, we found that an average of $73 \%$ of assumed supporting topics and $54 \%$ of other core topics chosen by all respondents at that institution.

These results support the anecdotal claim that upperdivision thermal physics content coverage varies both across institutions and between instructors at the same institution. It also makes the case, however, that there are some topics, namely, our assumed core topics, that all or most instructors prioritize in their upper-division thermal physics courses.

\section{ITEM DEVELOPMENT}

Evidence of content variability motivates the need for a flexible-format assessment in which instructors are able to customize assessment content for their particular class. First, however, we wanted to create a core set of items that could act as a baseline assessment for every upper-division thermal physics course. Thus, after analysis of survey results, we moved to developing items to target the topics identified on the survey, focusing on 10 topical areas chosen by most instructors.

The intent is for the final assessment to be composed of multiple-choice (MC) and coupled, multiple-response (CMR) items. CMR items are composed of a MC question followed with a prompt asking students to select one or more reasoning elements utilized to find the first answer [1]. CMR formats are ideal for our assessment because they provide insight into student reasoning (much like freeresponse items) while also allowing for online administration and more streamlined scoring that could be automated. For brevity, we will refer to MC and CMR items collectively as multiple-response (MR) items.

Initial item development took place in two broad stages: (1) developing assessment objectives for each of the 10 identified topical areas and (2) writing and refining assessment items based on those objectives. The full development 


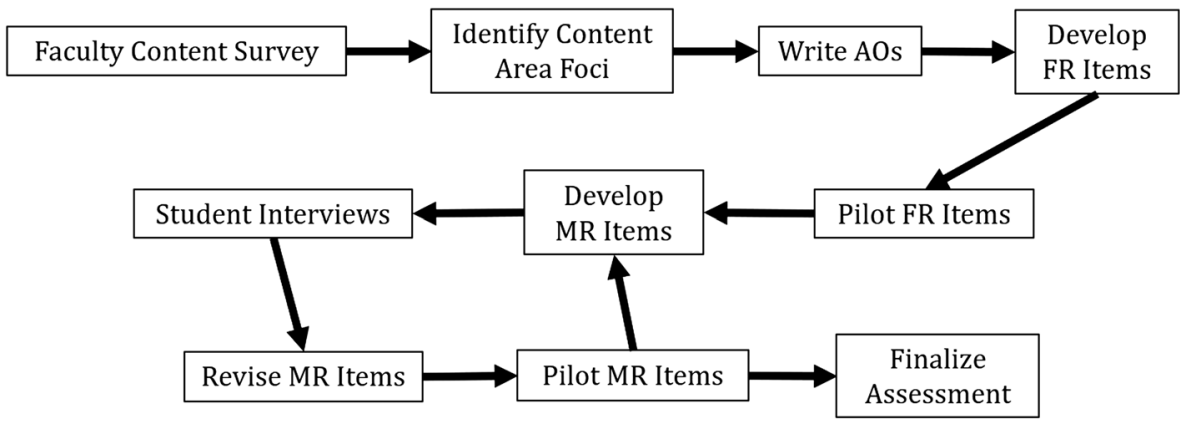

FIG. 4. The full development process of the assessment. After the content survey, key content areas are identified. Free-response (FR) items are then written, revised iteratively, then piloted. Using results of the FR pilot administration, multiple-response (MR), composed of both multiple-choice (MC) and coupled, multiple-response (CMR), items are developed. MR items are then tested for face validity in student interviews. After interviews, MR items are revised, and then piloted. After initial MR piloting, MR item development is revisited, involving item development, student interviews, and revision. Items are then piloted again, leading to a finalized assessment.

process is summarized in Fig. 4 and aligns with processes used for developing similar upper-division assessments [26]. We note that the MR items will be piloted at least twice before the assessment is finalized.

In this section, we describe the item development process, including assessment objective (Sec. IVA) and item (Sec. IV B) development, and end by presenting an example of the full process for a single item in statistical mechanics (Sec. IV C).

\section{A. Assessment objectives}

We developed assessment objectives to guide the writing of our assessment items. We intentionally call these objectives assessment objectives (AOs), as opposed to learning objectives, because they were specifically designed to guide development of assessment items. We do not intend for the objectives we wrote to span the full scope of objectives that may appear in upper-division thermodynamics, statistical mechanics, or thermal physics courses. Instead, they span the space of feasible, testable outcomes for the specific assessment we are developing.

We established two criteria for our assessment objectives: (1) they must collectively span the space of content areas identified as important based on faculty responses on the survey, and (2) they must be directly assessable in a MR format. When developing the objectives, we began by identifying concepts and turned these into actionable statements that could be assessed. For example, a concept would be something like "probability" or "Boltzmann factor," whereas an assessment objective would be "students can determine probabilities for thermodynamic systems using the Boltzmann factor." Here, the AO meets both criteria by expressing an assessable action students should be able to do with their content knowledge.

We began by developing AOs for core topics and supporting topics appearing with frequencies above $90 \%$ on the faculty content survey. Some topics that were similar but appeared in different areas on the survey, such as the 2nd law and entropy, were combined into a single topical area. In the end, we focused on developing AOs for the following topical areas (in alphabetical order): energy; engines and refrigerators; entropy and the 2nd law; equilibrium; the 1st law; gases; heat; statistical mechanics; temperature; and work.

From these topical areas, we created initial AOs. From these initial drafts, the three authors iterated and refined the objectives. After these authors were satisfied with the AO drafts, we distributed them to external reviewers. We received feedback on the AOs from two of these external reviewers. We then finalized the AOs based on reviewer comments. For example, a finalized $\mathrm{AO}$ that targeted equipartition and degrees of freedom was "Students can articulate that the internal energy of an ideal gas is determined by the number of degrees of freedom available to atoms or molecules comprising the gas system."

We drafted 57 AOs across the 10 identified topical areas, some of which had supporting objectives as well. Statistical mechanics had the most AOs (eleven). Some AOs overlapped across topical areas, but remained in the topical area that it more closely addressed. For example, some 1st law AOs contained both work and heat but stayed within the "1st law" topical area because they addressed heat and work only within the context of the 1st law, as opposed to in isolation. We documented crossover such as this in our notes for future reference.

\section{B. Assessment items}

We used the AOs to directly inform assessment item drafting. All AOs were addressed by at least one, and often more than one, drafted assessment item. In total, we drafted 86 potential assessment items. All initial items were free response or multiple choice with a prompt to explain reasoning. This was motivated by the need for attractive distractors to be included on the finalized MR assessment.

After drafting potential assessment items, we narrowed our focus on a subset of items that addressed the widest 
TABLE III. Number of items for each version of the free response thermal physics beta-assessment version.

\begin{tabular}{lccc}
\hline \hline & Anchor questions & Secondary set 1 & Secondary set 2 \\
\hline Version A & 6 (set i) & 6 & $\ldots$ \\
Version B & 6 (set ii) & $\ldots$ & 6 \\
Version C & 6 (set i) & 6 (reversed) & $\ldots$ \\
Version D & 6 (set ii) & $\ldots$ & 6 (reversed) \\
\hline \hline
\end{tabular}

range of AOs while resulting in a feasible number of items that we could commit to revising. We identified the most essential AOs from the pool of 57 written objectives, ensuring the shortlist spanned the 10 identified topical areas. We then identified 24 items that addressed the majority of these objectives. The next step was to pilot these items so they could be turned into MR items for the final assessment.

In developing pilot assessments for these 24 items, we had to strike a balance between collecting enough information about individual items to adequately inform developing them into MR items and having the piloted assessment be short enough to complete in the given time period (50 min). We split the assessment items into four groups of six items; two "anchor sets" and two "secondary sets." Anchor questions were questions that were likely to be on the finalized assessment, while questions composing the secondary sets were questions with more uncertainty related to their inclusion in the finalized assessment (e.g., they may prove to be too easy or too difficult to include in the final version).

We developed four beta-assessment versions, each containing one anchor set followed by one secondary set, making each beta-assessment version 12 items long. For two of the beta-assessment versions, the order of questions in the secondary sets was reversed so that, if students answered questions in order but ran out of time, we would still have a sufficient number of responses to every item. Each group of six questions was included on two betaassessment versions (outlined in Table III).

The beta-assessment versions were piloted as free response in an upper-division thermal physics course in a large physics department in Fall 2019. They were administered in-class during a $50 \mathrm{~min}$ period. We received responses from 61 students, the majority of whom selfidentified as white (77\%), male (70\%), and seniors $(87 \%)$. The completion times for students ranged between 18 and $50 \mathrm{~min}$, taking an average of $31 \mathrm{~min}$ overall.

After piloting, student responses were analyzed and coded based on what directly emerged from what students wrote [49]. We illustrate this process with an example: In response to a question regarding the difference between heat and temperature, responses such as "heat is a measure of how much energy is transferred to or from a system" and "heat only exists as a transfer of energy" were both coded as "heat as energy transfer." This example code resulted in
MR selections such as "heat is a flow of thermal energy" and "heat is a quantity exchanged between systems" for CMR items related to heat. For all FR items, responses and corresponding codes were used to inform the rewriting and revising of items to convert them into MC or CMR formats.

Once we reached MC and CMR formats, we conducted five think-aloud interviews with students to verify the items were being interpreted in the way they were intended to [50-52]. Small revisions were made based on the outcomes of interviews. For example, one question related to the equipartition theorem asked When does the equipartition theorem hold? For the question, students would select any situation that the equipartition theorem holds for (e.g., selecting "when the system is a solid" because the equipartition theorem does apply for Einstein solids) instead of requirements that must hold for the equipartition theorem to be applied, as the question originally intended. Thus, the prompt was changed to Which of the following must be true in order for the equipartition theorem to be used for a given system?

The following section outlines our process for a single assessment item from the statistical mechanics topical area.

\section{An example in statistical mechanics}

A total of seven statistical mechanics questions were piloted across the four beta-assessment versions, with 3-4 statistical mechanics items appearing on each version. In this section, we focus on one of these items to demonstrate the full cycle of item development, from AOs to its finalized CMR version to be used in the implementation of the first set of MR items.

\section{Developing the assessment objectives}

From the content survey, we identified several content goals for statistical mechanics. The example we highlight here covers the following content goals: probability, counting, the Boltzmann factor, and the partition function. From these content goals, we developed the following initial AOs:

1. Students can determine probabilities.

(a) Students can determine probabilities for simple systems using counting.

(b) Students can determine probabilities for thermodynamic systems using the Boltzmann or Gibbs factor.

2. Students can deduce state probability from a partition function.

3. Students can compare probabilities of states.

(a) Students can compare probabilities for simple systems using counting.

(b) Students can determine and compare probabilities for a system after an energy shift using the partition function.

These AOs all have action words (e.g., determine, deduce, and compare) and could be directly assessed with 
A particle is in system A, with the energy of each available state indicated on the left. The system undergoes a process such that the energy of the states is shifted by $\Delta \mathrm{E}>0$ (referred to as system B).

(i) How does the partition function change due to the energy shift?

a) increase

b) decrease

c) remains the same

Explain your reasoning.

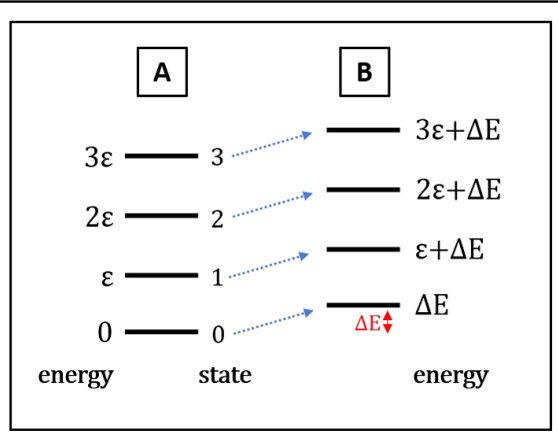

(ii) How does the probability of the particle being in state 2 change due to the energy shift?

a) increase

b) decrease

c) remains the same

Explain your reasoning.

(iii) How does the ratio of probabilities of being in states 1 and 2 compare between $A$ and $B$ ? That is, how does

$\mathrm{P}_{\mathrm{A}}(1) / \mathrm{P}_{\mathrm{A}}(2)$ compare to $\mathrm{P}_{\mathrm{B}}(1) / \mathrm{P}_{\mathrm{B}}(2)$ ?

a) less than

b) greater than

c) equal

Explain your reasoning.

FIG. 5. A statistical mechanics free-response item piloted in the Fall 2019 beta-assessment version. The item is considered to be freeresponse despite its inclusion of multiple-choice options because of the prompt to explain student reasoning.

a well-written assessment item. After these were finalized, they underwent review from two experts familiar with teaching and researching upper-division thermal physics. With reviewer feedback in mind, the AOs from above were modified and combined into the following singular AO:

Students can determine and compare probabilities of states.

(a) Students can determine and compare probabilities of simple systems using counting.

(b) Students can determine and compare probabilities for a thermodynamic system using the Boltzmann factor.

(c) Students can determine and compare probabilities for a thermodynamic system after an energy shift using the Boltzmann factor.

\section{Developing the beta-assessment item}

After articulating this $\mathrm{AO}$, we wrote an item draft inspired by a problem developed for a Boltzmann factor tutorial [23], as shown in Fig. 5. The item asks students about changes in the partition function and state probabilities after an atomic system undergoes an energy shift of $\Delta E$ that takes the system from system $A$ to system $B .{ }^{5}$ This

\footnotetext{
${ }^{5}$ As described in a later section, we changed the wording of the problem to ask "what happens when the system goes from configuration A to configuration B" to address potential confusion about the number of systems discussed in the problem (i.e., there is only one system, but it could be interpreted as two separate systems).
}

item was designed to directly address parts (b) and (c) of the finalized AO discussed above.

\section{Piloting the beta-assessment item}

The item shown in Fig. 5 was included in versions A and D of the beta-assessment item (see Table III) and 29 student responses were collected. Table IV summarizes responses to the $\mathrm{MC}$ portions of the item presented in Fig. 5. For (i), incorrect responses were split somewhat evenly between increase, remains the same, and a written "I don't know" (indicated in Table IV as "unsure/no answer"). For (ii), decrease was a popular incorrect answer, provided by $31 \%$ of respondents. Justifications for decrease were often related the negative exponent in the Boltzmann factor that appears in the numerator of the probability function. Here, students did not identify the identical change in the partition

TABLE IV. Response frequency for the multiple-choice portion of the assessment item presented in Fig. 5. Bolded responses indicate the correct response.

\begin{tabular}{lcccc}
\hline \hline & & \multicolumn{3}{c}{ Remains } \\
& Increase & Decrease & the same & Unsure/No answer \\
\hline (i) & $21 \%$ & $\mathbf{4 5 \%}$ & $17 \%$ & $17 \%$ \\
(ii) & $14 \%$ & $31 \%$ & $\mathbf{3 8 \%}$ & $17 \%$ \\
\hline & Less than & Greater than & Equal to & Unsure/No answer \\
(iii) & $0 \%$ & $17 \%$ & $\mathbf{7 2 \%}$ & $10 \%$ \\
\hline \hline
\end{tabular}


A particle is in configuration A, with the energy of each available state indicated on the left. The system undergoes a process such that the energy of the states is shifted by $\Delta \mathrm{E}>0$ (referred to as configuration $\mathrm{B}$ ). (See figure.)

(i) How does the partition function change due to the energy shift?

The partition function...
a) increases
b) decreases
c) remains the same

because... (select all that support your response above)

$\square$ there are the same number of states before and after the energy shift

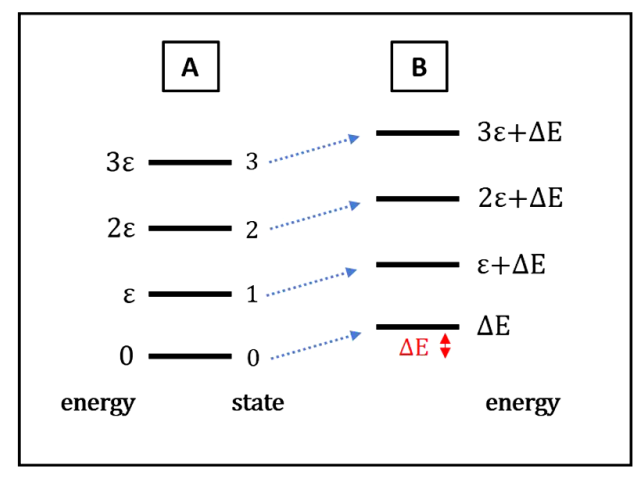

$\square$ the partition function does not depend on the value of the energy level

$\square$ each term in the partition function gains an $e^{\Delta E / k_{B} T}$ factor

$\square$ each term in the partition function gains an $e^{-\Delta E / k_{B} T}$ factor

$\square$ the states have more energy

$\square$ the particles in configuration B would have more energy than particles in configuration A

other:

(ii) How does the probability of a particle being in state $2, \mathcal{P}(2)$, change due to the energy shift?

$\mathcal{P}(2) \ldots$
a) increases
b) decreases
c) remains the same

because... (select all that support your response above)

$\square$ the particle is more excited

$\square$ the Boltzmann factor increased due to the energy shift

$\square$ the Boltzmann factor decreased due to the energy shift

$\square$ the partition function increased due to the energy shift

$\square$ the partition function decreased due to the energy shift

$\square$ the Boltzmann factor and partition function changed by the same factor

$\square$ the Boltzmann factor changed by a greater factor than the partition function

$\square$ the partition function changed by a greater factor than the Boltzmann factor

$\square$ there are the same number of possible states before and after the energy shift

$\square$ other:

(iii) How does the ratio of probabilities of being in states 1 and 2 compare between $A$ and $\mathrm{B}$ ? That is, how does $\mathcal{P}_{\mathrm{A}}(1) / \mathcal{P}_{\mathrm{A}}(2)$ compare to $\mathcal{P}_{\mathrm{B}}(1) / \mathcal{P}_{\mathrm{B}}(2)$ ?

The ratio $\mathcal{P}_{\mathrm{A}}(1) / \mathcal{P}_{\mathrm{A}}(2)$ is...

a) less than

b) greater than

c) equal to

$\mathcal{P}_{\mathrm{B}}(1) / \mathcal{P}_{\mathrm{B}}(2)$ because... (select all that support your response above)

probability increases with increasing energy

probability decreases with increasing energy

state 2 is more probable in configuration A than configuration $B$

state 2 is more probable in configuration B than configuration A

$\square$ each probability changes by the same factor going from configuration $A$ to $B$

$\square$ the difference in energy levels is the same in both systems

$\square$ the ground state energy changes the same amount as other states' energies

other:

FIG. 6. A statistical mechanics coupled, multiple-response item developed based on responses to the free-response version of the same item piloted in the Fall 2019 beta-assessment version (see Fig. 5).

function due to the energy shift, which appears in the denominator. Most students answered (iii) correctly, but there was a variety of reasoning used to justify their responses. Some utilized similar reasoning from (i) and (ii), saying that everything changed by the same factor, whereas others relied on physical reasoning without invoking mathematics (e.g., there is the same spacing between the energy levels in both systems). 


\section{Refining the item: Coupled, multiple response}

After analyzing student responses to the free-response (FR) item presented in Fig. 5, the item was transformed into a CMR format [1]. One change was made to the prompt after the analysis: "system A" and "system B" were changed to "configuration A" and "configuration B". This was done to mitigate any confusion that may arise for students. Configuration B is the same system as configuration A, just after an energy shift; with the original wording, the two diagrams could potentially be interpreted by students as two independent systems.

Student responses to the "explain your reasoning" prompts for each portion of the FR item were used to create reasoning elements for the CMR version. The research team also created additional reasoning elements to capture possible reasoning not expressed in students FR responses. Reasoning elements are provided to students in the CMR item as choices to support their answer to the MC prompt.

Figure 6 shows the finalized CMR version of the item presented in Fig. 5. The expectation for the CMR item would be for students to select one response for the $\mathrm{MC}$ question (increase, decrease, or remains the same), then select as many reasoning elements as needed in the followup question that supports their choice. The selected reasoning elements give insight into particular student difficulties or understandings. We note that the lists of reasoning elements provided in the item presented in Fig. 6 are relatively long and one may hypothesize the length of these lists exhaust students or influence response patterns. However, research shows that long lists of reasoning elements in CMR items do not significantly change response patterns or validation statistics when comparing CMR items to the FR versions used for development of those items [1,53].

As an example of possible insight provided from reasoning elements, if a student incorrectly said the probability decreased and selected "the Boltzmann factor decreased due to the energy shift" but did not select anything related to the partition function or the relationship between the partition function and Boltzmann factor in determining probability, this would indicate the student likely does know that probability is directly proportional to the Boltzmann factor, but did not consider the partition function's contribution to the probability as well. Each possible MC option has some combination of reasoning elements provided in the follow up that could support the MC selection, and students are able to form their own reasoning from the provided reasoning elements. An "other" option is provided as well to solicit other students reasoning elements that do not appear in the provided list of options inspired by FR explanations during the initial pilot. This was done in part to offset the limitation of piloting the FR version at only one institution. After developing the CMR item, it was piloted in five think-aloud interviews to verify it was being interpreted as intended. This particular item did not change after interviews, though some other items did. We note that no students indicated in the interviews that the lists of reasoning elements were too long for them to comprehend effectively.

\section{FUTURE WORK}

Of the piloted FR items, 14 were converted into CMR items. The other 10 items were made into $\mathrm{MC}(N=6)$, discarded $(N=1)$, or reserved for future work $(N=3)$. Multiple-choice items were developed from FR items that did not have diverse reasoning elements emerge or had answers that could indicate the approach students utilized without explaining reasoning. We chose to discard one item based on difficulty for students (i.e., few were able to answer it correctly or make a solid attempt at a solution). The remaining 3 items addressed scientific practices and were not revised during the initial revision cycle. Those items will be revisited in the future.

The next step in developing the assessment will be to pilot the MR items revised from the FR beta-assessment item in an online format. The online administration is motivated by allowing more institutions to be used for piloting and streamlined analysis. We have little concern about students potentially using online resources to perform better on the assessment, as answers to the items will not be found easily via online search and a recent study shows certain online behaviors that may be linked to using internet resources do not appear to meaningfully influence average student performance on similar research-based assessments [54]. Additionally, we advise, in line with best practices from the assessment literature $[55,56]$, that instructors only provide participation credit to students for completion of the assessment (as opposed to assigning scores based on correctness). This lower-stakes administration may minimize student motivation to use outside resources. We anticipate having on the order of 150 student responses from this initial MR pilot. All piloted CMR items will have a text box "other" reasoning element option to solicit reasoning elements that did not emerge during the FR piloting.

After piloting the MR items, we will perform initial CTT and Rasch analyses. Based on outcomes of these analyses, items will be revised or discarded. We will then pilot the newly revised MR items with another, large-scale implementation in an online format. We will compute final validation statistics, and then finalize the upper-division thermal physics assessment. This assessment and the results of the faculty content survey will lay the foundation for a similar assessment that will aim to allow for flexible content coverage (i.e., allow instructors to select what content is included in the assessment they administer), which will be the focus of future work. 


\section{CONCLUSIONS}

Here, we presented the process we have used to take initial steps in developing a multiple-response, upperdivision thermal physics assessment that includes both multiple-choice and coupled, multiple-response items. This process is distinct from previous approaches in that it prioritizes faculty perspectives. Additionally, in order for this assessment to be useful broadly, we deliberately collected data from institutions that serve a wide range of student populations. We recommend other researchers interested in making widely available upper-division materials utilize similar methods in collecting input from a wide range of institutions to inform their work.

We began by administering a faculty content survey to solicit content priorities in upper-division thermal physics courses. We used results from this survey to guide development of assessment items, which were administered in a free-response format in an upper-division thermal physics course before being transformed into multiple-response (MR) items. The MR items are, as of the submission of this paper, being piloted in an online format. We will use the results of these pilot assessments to compute initial validation statistics using statistical techniques beyond the standard analyses used with classical test theory. We will revise items based on these analyses and then pilot them again before the assessment is finalized.
Our data also suggest important considerations for researchers and instructors interested in curricular materials and assessment development for upper-division thermal physics. Despite the demonstrated content variability within thermal physics, our results point to content foci, scientific practices, and reference texts that can act as baselines for upper-division thermal physics materials that have potential to serve a broad range of institutions and instructors.

The results presented here will lay the groundwork for development of an upper-division thermal physics assessment that allows for flexible content coverage. The methodology we used can be reproduced for investigations of the scope of other upper-division physics courses.

\section{ACKNOWLEDGMENTS}

We thank S. Pollock, M. Dubson, D. Roundy, J. Thompson, and all focus group participants for their input in this work. We also thank the department chairs who distributed the content survey and the faculty who completed it. We are grateful for their time. This work was supported by funding from the Center for STEM Learning and the Department of Physics at the University of Colorado Boulder.
[1] B. R. Wilcox and S. J. Pollock, Coupled multiple-response versus free-response conceptual assessment: An example from upper-division physics, Phys. Rev. ST Phys. Educ. Res. 10, 020124 (2014).

[2] NGSS Lead States, Next Generation Science Standards: For States, By States (National Academies Press, Washington, DC, 2013).

[3] D. Hestenes, M. Wells, and G. Swackhamer, Force Concept Inventory, Phys. Teach. 30, 141 (1992).

[4] A. Savinainen and P. Scott, The Force Concept Inventory: A tool for monitoring student learning, Phys. Educ. 37, 45 (2002).

[5] B. W. Dreyfus, B. D. Geller, D. E. Meltzer, and V. Sawtelle, Resource letter TTSM-1: Teaching thermodynamics and statistical mechanics in introductory physics, chemistry, and biology, Am. J. Phys. 83, 5 (2015).

[6] J. W. Clark, J. R. Thompson, and D. B. Mountcastle, Comparing student conceptual understanding of thermodynamics in physics and engineering, AIP Conf. Proc. 1513, 102 (2013).

[7] S. Yeo and M. Zadnik, Introductory thermal concept evaluation: Assessing students' understanding, Phys. Teach. 39, 496 (2001).
[8] H.-E. Chu, D. F. Treagust, S. Yeo, and M. Zadnik, Evaluation of students' understanding of thermal concepts in everyday contexts, Int. J. Sci. Educ. 34, 1509 (2012).

[9] C. Tanahoung, R. Chitaree, C. Soankwan, M. Sharma, and I. Johnston, Surveying Thai and Sydney introductory physics students understandings of heat and temperature, in Proceedings of The Australian Conference on Science and Mathematics Education (formerly UniServe Science Conference), Sydney, Australia (2012).

[10] P. Wattanakasiwich, P. Taleab, M. D. Sharma, and I. D. Johnston, Construction and implementation of a conceptual survey in thermodynamics, Int. J. Innovation Sci. Math. Educ. (formerly CAL-laborate International) 21, 29 (2013), https://openjournals.library.sydney.edu.au/index .php/CAL/article/view/6459.

[11] B. Brown, Developing and assessing research-based tools for teaching quantum mechanics and thermodynamics, Ph.D. thesis, University of Pittsburgh, Pittsburgh, Pennsylvania, 2015.

[12] P. G. Jasien and G. E. Oberem, Understanding of elementary concepts in heat and temperature among college students and K-12 teachers, J. Chem. Educ. 79, 889 (2002). 
[13] M. Sözbilir, A review of selected literature on students' misconceptions of heat and temperature, Boğaziçi Univ. J. Educ. 20, 25 (2003), https://www.researchgate.net/ publication/228768141_A_review_of_selected_literature_ on_students\%27_misconceptions_of_heat_and_temperature.

[14] A. A. Alwan, Misconception of heat and temperature among physics students, Procedia-Soc. Behav. Sci. 12, 600 (2011).

[15] T. I. Smith, W. M. Christensen, D. B. Mountcastle, and J. R. Thompson, Identifying student difficulties with entropy, heat engines, and the carnot cycle, Phys. Rev. ST Phys. Educ. Res. 11, 020116 (2015).

[16] M. E. Loverude, Identifying student resources in reasoning about entropy and the approach to thermal equilibrium, Phys. Rev. ST Phys. Educ. Res. 11, 020118 (2015).

[17] M. Sozbilir, What students understand from entropy?: A review of selected literature, J. Baltic Sci. Educ. 2, 21 (2003), http://www.scientiasocialis.lt/jbse/?q=node/51.

[18] W. M. Christensen, D. E. Meltzer, and C. Ogilvie, Student ideas regarding entropy and the second law of thermodynamics in an introductory physics course, Am. J. Phys. 77, 907 (2009).

[19] C. H. Kautz, P. R. Heron, M. E. Loverude, and L. C. McDermott, Student understanding of the ideal gas law, Part I: A macroscopic perspective, Am. J. Phys. 73, 1055 (2005).

[20] C. H. Kautz, P. R. Heron, P. S. Shaffer, and L. C. McDermott, Student understanding of the ideal gas law, Part II: A microscopic perspective, Am. J. Phys. 73, 1064 (2005).

[21] D. E. Meltzer, Investigation of students reasoning regarding heat, work, and the first law of thermodynamics in an introductory calculus-based general physics course, Am. J. Phys. 72, 1432 (2004).

[22] M. E. Loverude, C. H. Kautz, and P. R. Heron, Student understanding of the first law of thermodynamics: Relating work to the adiabatic compression of an ideal gas, Am. J. Phys. 70, 137 (2002).

[23] T. I. Smith, D. B. Mountcastle, and J. R. Thompson, Student understanding of the Boltzmann factor, Phys. Rev. ST Phys. Educ. Res. 11, 020123 (2015).

[24] T. I. Smith, J. R. Thompson, and D. B. Mountcastle, Student understanding of Taylor series expansions in statistical mechanics, Phys. Rev. ST Phys. Educ. Res. 9, 020110 (2013).

[25] T. A. Moore and D. V. Schroeder, A different approach to introducing statistical mechanics, Am. J. Phys. 65, 26 (1997).

[26] B. R. Wilcox, M. D. Caballero, C. Baily, H. Sadaghiani, S. V. Chasteen, Q. X. Ryan, and S. J. Pollock, Development and uses of upper-division conceptual assessments, Phys. Rev. ST Phys. Educ. Res. 11, 020115 (2015).

[27] R. Hambleton and J. Rogers, Item bias review, Pract. Assess. Res. Eval. 4, 6 (1994).

[28] P. V. Engelhardt, An introduction to classical test theory as applied to conceptual multiple-choice tests, in Getting Started in PER, Reviews in PER, edited by C. Henderson and K. A. Harper (American Association of Physics Teachers, College Park, MD, 2009), Vol. 2.

[29] L. E. Kost, S. J. Pollock, and N. D. Finkelstein, Characterizing the gender gap in introductory physics, Phys. Rev. ST Phys. Educ. Res. 5, 010101 (2009).
[30] S. J. Pollock, N. D. Finkelstein, and L. E. Kost, Reducing the gender gap in the physics classroom: How sufficient is interactive engagement?, Phys. Rev. ST Phys. Educ. Res. 3, 010107 (2007).

[31] M. Lorenzo, C. H. Crouch, and E. Mazur, Reducing the gender gap in the physics classroom, Am. J. Phys. 74, 118 (2006).

[32] R. R. Hake, Relationship of individual student normalized learning gains in mechanics with gender, high-school physics, and pretest scores on mathematics and spatial visualization, in Proceedings of the 2002 Physics Education Research Conference, Boise, Idaho, Vol. 8 (2002) pp. 1-14.

[33] National Science Foundation, National Center for Science and Engineering Statistics, Women, Minorities, and Persons with Disabilities in Science and Engineering, Arlington, VA (National Science Foundation, Washington, DC, 2017), Special Report NSF 17-310.

[34] G. Rasch, Probabilistic Models for Some Intelligence and Attainment Tests (Danish Institute for Educational Research, Copenhagen, 1980).

[35] R. Baierlein, Thermal Physics (Cambridge University Press, Cambridge, England, 1999).

[36] A. Carter, Classical and Statistical Thermodynamics (Prentice Hall, Englewood Cliffs, NJ, 2001).

[37] C. Kittel and H. Kroemer, Thermal Physics (W. H. Freeman \& Co., San Francisco, CA, 1980).

[38] F. Sears and G. Salinger, Thermodynamics, Kinetic Theory and Statistical Thermodynamics (Addison-Wesley Publishing Co., Reading, MA, 1975).

[39] D. V. Schroeder, An Introduction to Thermal Physics (Addison Wesley, Reading, MA, 1999).

[40] M. Zemansky and D. Dittman, Heat and Thermodynamics (McGraw-Hill, New York, NY, 1997).

[41] See Supplemental Material at http://link.aps.org/ supplemental/10.1103/PhysRevPhysEducRes.16.020113 for frequencies of other core topics and their supporting topics.

[42] American Physical Society, Top Educators, https://www .aps.org/programs/education/statistics/topproducers.cfm (2019).

[43] American Physical Society, 2017 Physics DegreeGranting Minority-Serving Institutions, https://www.aps .org/programs/education/statistics/upload/List-HBCUsBSIs-HSIs-2017.pdf (2017).

[44] R. Ivie and K. Stowe, Women in Physics, 2000, in AIP Report (American Institute of Physics, New York, 2000).

[45] PhysNet: The physics departments and documents network, Physics Departments in the United States of America, http://de.physnet.net/PhysNet/us.html (2013).

[46] The Carnegie classification of Institutions of Higher Education, http://carnegieclassifications.iu.edu/ (2018).

[47] F. Reif, Fundamentals of Statistical and Thermal Physics (Waveland Press, Long Grove, IL, 2009).

[48] National Research Council, A Framework for K-12 Science Education: Practices, Crosscutting Concepts, and Core Ideas (National Academies Press, Washington, DC, 2012).

[49] S. E. Stemler, Content analysis, emerging trends in the social and behavioral sciences: An interdisciplinary, searchable, and linkable resource, 1 (John Wiley \& Sons, 
Inc., New York, 2015), https://doi.org/10.1002/ 9781118900772.etrds0053.

[50] K. A. Ericsson and H. A. Simon, Verbal reports as data, Psychol. Rev. 87, 215 (1980).

[51] R. Jääskeläinen, Think-aloud protocol, Handb. Transl. Studies 1, 371 (2010).

[52] J. W. Cresswell, Research Design: Qualitative, Quantitative, and Mixed Methods Approaches, 4th ed. (SAGE Publications, Inc., Thousand Oaks, CA, 2014), pp. 183-213.

[53] B. R. Wilcox and S. J. Pollock, Validation and analysis of the coupled multiple response Colorado upper-division electrostatics diagnostic, Phys. Rev. ST Phys. Educ. Res. 11, 020130 (2015).

[54] B. R. Wilcox and S. J. Pollock, Investigating students behavior and performance in online conceptual assessment, Phys. Rev. Phys. Educ. Res. 15, 020145 (2019).

[55] L. Ding, N. W. Reay, A. Lee, and L. Bao, Effects of testing conditions on conceptual survey results, Phys. Rev. Phys. Educ. Res. 4, 010112 (2008).

[56] C. Henderson, Common concerns about the Force Concept Inventory, Phys. Teach. 40, 542 (2002). 